\title{
Las luchas indígenas por el derecho fundamental a la salud propio e intercultural en Colombia
}

\author{
Indigenous struggles for an own and intercultural fundamental right \\ to health in Colombia
}

Jaime Hernán Urrego-Rodríguez

DOI: $10.1590 / 0103-110420205106$

\begin{abstract}
RESUMEN El presente artículo hace una aproximación a las luchas de los pueblos indígenas en Colombia, particularmente aquellos pertenecientes al Consejo Regional Indígena del Cauca (Cric), por la materialización del derecho fundamental a la salud, a lo largo de cinco períodos históricos entre 1971 y 2019. Este artículo presenta las características de la disputa política con el Estado, la transformación contenciosa de la política de salud indígena, direccionada desde el estado, y de la política indígena de salud, promovida desde las luchas de los pueblos indígenas. Ambas políticasse expresan en enfoques, normas e instituciones en un contexto de violencia, discriminación y racismo; en la permanente tensión entre un Sistema General de Seguridad Social en Salud (SGSSS) oficial, con una lógica de mercado y rentabilidad económica, y un Sistema de Salud Indígena, Propio e Intercultural (Sispi) de carácter colectivo y público, que desafía las asimetrías de poderes y saberes entre el modelo hegemónico de desarrollo, la medicina occidental y democracia restringida, orientado hacia el buen vivir, la sabiduría ancestral y la acción política colectiva basada en la ley de origen, el derecho mayor y el derecho propio de los pueblos indígenas.
\end{abstract}

PALABRAS CLAVE Derecho a la salud. Grupos de población. Política de salud.

ABSTRACT This article provides an approximation to the struggles of indigenous peoples in Colombia for the materialization of the fundamental right to health. The study emphasizes the case of the Regional Indigenous Cauca's Council (Cric). It reviews five historical periods between 1971 and 2019. While doing so, the paper presents the characteristics of the political dispute between Cric and the State, the contentious transformation of the indigenous health policy, and the indigenous health policy expressed in approaches, norms, and institutions in a context of violence, discrimination, and racism. It highlights the permanent tension between a General System of Social Security (SGSSS), embodying the logic of a market and economic profit ability and an indigenous Health System Integrating Own and Intercultural (Sispi) of collective and public nature that challenges the asymmetries of power and knowledge among the hegemonic model of development, western medicine, and restricted democracy. In contrast to this latter, the indigenous health system is one oriented towards the good living, the ancestral wisdom, and the collective political action based on the law of origin, the greater right and the own right of the indigenous peoples.

KEYWORDS Right to health. Population groups. Health policy.

1 Universidade Nacional de Colombia (Unal) - Bogota (DC), Colombia.

finseya5@gmail.com 


\section{Introducción}

Los pueblos indígenas agrupados en el Consejo Regional Indígena del Cauca (Cric) con gran tradición de lucha, vienen construyendo alternativas y resistiendo en medio de la violencia y el racismo estructural, los embates de un modelo de desarrollo depredador de la vida, del territorio y de una salud integral comprendida como equilibrio de los sujetos colectivos de acuerdo a sus cosmovisiones, saberes y formas de gobierno diversos. Resulta relevante, por lo tanto, comprender la disputa política por la configuración contenciosa de la política de salud indígena, direccionada desde el Estado y de la política indígena de salud, surgida desde las luchas de los pueblos indígenas en la perspectiva de construcción del buen vivir desafiando la institucionalidad y la medicina hegemónica.

De este modo, se presentan los resultados de la investigación de la tesis doctoral en Salud Pública sobre la configuración contenciosa entre las políticas de salud indígena que asume el Estado y aquellas que van estructurando los pueblos indígenas, fundamentalmente del Cauca, entre 1971 y 2017. Para este fin, se analizarán cinco periodos: el surgimiento y desarrollo inicial del Cric, su plataforma de lucha incluida la salud y primeros instrumentos de política de salud indígena del Estado, (19711990); el proceso constituyente (1990-1991); la reforma neoliberal e inconsulta del Sistema General de Salud y Seguridad Social (SGSSS)/ Ley 100; la resistencia del Cric subordinante de lo indígena al SGSSS, incluida la creación de empresas administradoras y prestadoras indígenas de salud y la Ley 691, (1993-2001); las adecuaciones socioculturales contenciosas a un SGSSS en crisis y emergencia de una propuesta de salud de los pueblos indígenas (2002-2010) y; el Sistema Indígena de Salud Propio e Intercultural (Sispi) como política indígena de salud en disputa con el SGSSS y como referencia autonómica en tiempos de construcción de paz (2011-2017).

Se observará en estos cinco periodos, los hitos en la construcción de la política pública a nivel del Estado y de los pueblos indígenas en los que se reflejan las disputas de poderes y saberes sobre gobernabilidad y modelos de salud en medio de movilizaciones, negociaciones y hechos políticos. Con esto se espera contribuir al debate sobre el derecho fundamental a la salud intercultural de los pueblos indígenas.

\section{Material y métodos}

La presente investigación rastrea la emergencia del sujeto indígena en el devenir socio-histórico y cultural de sus luchas en Colombia, a partir del surgimiento del Cric. Su emergencia refleja las dinámicas asimétricas del poder y sus consecuencias clasificatorias y racializadoras de origen e impronta colonial, en el marco del sistema mundo capitalista ${ }^{1}$ y de los discursos de la otredad ${ }^{2}$. En particular mediante del análisis cualitativo de fuentes primarias como documentos normativos, técnicos, políticos, prensa indígena, local y nacional, y entrevista de 12 actores clave participantes del proceso, se da cuenta de cuáles han sido los principales hitos y por qué, en la transformación normativa, programática e institucional como expresiones concretas y contenciosas de las políticas de salud tanto desde el ámbito estatal como del sujeto político indígena en materia de salud e interculturalidad surgidas en la disputas de poderes y saberes entre 1971 y 2017.

\section{Resultados y discusión}

En Colombia, según el Censo de 2005 la población indígena es de 1.392 .623 personas: un $3,43 \%$ de la población total, pertenecientes a 102 pueblos indígenas que habitan 713 resguardos (territorios gobernados por autoridades indígenas) ${ }^{3}$. En el Cric confluyen $280 \mathrm{mil}$ indígenas (20\% de la población del departamento del Cauca, sur occidente colombiano) de 10 pueblos: Nasa,Totoró, Misak, Yanacona, Kokonuco, Embera, Embera Siapidara, Inga, 
Ampiule y Kishu, gobernados por 125 cabildos (Autoridades Político Administrativas indígenas) ${ }^{4}$. El pueblo Nasa, mayoritario dentro del Cric, ha liderado históricamente procesos de resistencia y lucha por la tierra y la pervivencia cultural. En 1890 bajo el Estado clerical, se expidió la Ley 89 que establecía:

La legislación general de la República no regirá entre los salvajes que vayan reduciéndose a la vida civilizada por medio de Misiones. En consecuencia, el Gobierno de acuerdo con la Autoridad eclesiástica, determinará la manera como esas incipientes sociedades deban ser gobernadas ${ }^{\mathbf{5}(1)}$.

No obstante, el corte racista y colonial de la norma, esta reconocía los resguardos y la autoridad del cabildo,por lo que se mantuvo la cruzada de terratenientes, iglesia y colonos contra los territorios indígenas. La arremetida fue enfrentada bajo el liderazgo del Nasa Manuel Quintín Lame mediante el levantamiento indígena del Cauca (19141916) y una serie de luchas hasta los años 60 fundamentadas en una plataforma de recuperación y ampliación de resguardos, no pago de terraje (explotaciónservilque permite al indígena trabajar una porción de tierra sin remuneración), defensa de la lengua, la historia y las costumbres y la formación de profesores indígenas ${ }^{6}$.

En 1958, las élites políticas y económicas pactan el Frente Nacional como instrumento de superación de un conflicto con epicentro en la propiedad de la tierra, cuyo diseño excluyente, profundizó la ya existente desigualdad social, llevó a una nueva fase de violencia política en el país. En medio de las luchas obreras, estudiantiles y campesinas por la transformación de la sociedad, el sujeto político indígena inmerso en la causa popular emerge diferenciado, desafiando el régimen político y su impronta colonial un pensamiento propio sobre territorio, gobierno y cultura, a partir de las luchas por la recuperación de tierras.

\section{1-1990: Las luchas del Cric, de la invisibilidad a la emergencia}

Durante los años 70, aún culminado el Frente Nacional, las élites continuaron en el poder, mediante fraudes electorales, el uso de la violencia y de los estados de excepción para enfrentar la crisis económica y el auge de las luchas sociales entre el 75 y el $90^{7}$.

En 1971 se celebra el primer congreso indígena del Cauca y del país, en el que nace el Cric bajo la consigna de Unidad, Tierra y Cultura. Allí se retoma la plataforma de Quintín Lame, salvo por el rechazo al racismo de la Ley 89 en lo concerniente a:

[...] la parte que trata de la minoría de edad ya que somos colombianos y ciudadanos de la República. [E incluir la] participación del sector indígena en la modificación de esas leyes ya que somos los que conocemos nuestros problemas y sus soluciones ${ }^{8(11)}$.

Con el nacimiento del Cric aumentan las recuperaciones de tierras y el ánimo por la unidad que llevó a realización del segundo congreso, en septiembre del mismo año, en el que se define inconveniente modificar la Ley 89 pues como señala Morales: "[...] esa ley autoriza la creación de resguardos y la creación de los cabildos, como autoridad máxima entre las comunidades indígenas [...]"9(138). Esto evidencia que en las luchas del Crich ay una impronta por controvertir y utilizar lo existente como forma de acumular poder desde lo propio, convirtiéndose en un sujeto político desafiante que se articula con otros movimientos sociales e impulsa la creación de organizaciones indígenas por todo el país.

Durante los años 70 se realizaron tres congresos más. En ellos aparecen como pilares la política de salud y la lucha por la tierra. Allí, el sentido otorgado al concepto de tierra es el del territorio para la cultura y la vida, en el que la armonía - salud para los pueblos indígenas-, es mantenida o restablecida por la medicina tradicional, según se orienta en dichos congresos 
[...] a) Que entre ellos discutan su ciencia y recuerden lo que han olvidado; que unan sus fuerzas para trabajar más efectivamente. b) Que con sus conocimientos nos colaboren en la preparación de nuestras luchas: advirtiendo los peligros, previniendo los males, protegiendo los compañeros [...] Los médicos instruirán a los Cabildos sobre los peligros y problemas y aconsejarán sobre la buena conducta de los miembros de los Cabildos como representantes de la autoridad indígena. Pedirles a los médicos que enseñen la botánica a la comunidad $[\ldots]^{10(44)}$.

Obsérvese cómo la promoción de la medicina tradicional emerge de una política de salud propia es decir desde la organización y según su cosmovisión, que surge desde el ámbito de la educación y la cultura; que se liga a la protección del territorio y la vida y la recuperación de tierras"1. Otro pilar es el fortalecimiento del cabildo como autoridad político-administrativa que,orientado por la medicina tradicional y la comunidad,propicia el equilibrio entre personas, familias, naturaleza y territorio. Se inicia así la construcción del gobierno en salud como componente de la política indígena.

En 1977, el Cric crea una red de más de 100 Agentes Comunitarios Indígenas Bilingües que impulsan la organización en salud, resuelven problemas básicos con botiquines (medicamentos y plantas medicinales) y protegen con los médicos tradicionales las luchas por la tierra. En un cursillo para esta red en Paniquitá, 27 delegaciones de diferentes puntos del Cauca "[...] que ya han organizado botiquines o se van a organizar, por lo general dependientes de las tiendas comunales, Empresas Comunitarias [...]"10(5), evidencian que el Cric crea un tejido autogestionario propio e intercultural que integra iniciativas económicas, de educación y salud para enfrentar la desatención estatal:

[...] son numerosos los compañeros que diariamente mueren por diarreas, gastroenteritis, gripas, hemorragias, etc. y en la mayoría de los casos por no haberles prestado un servicio a tiempo pues casi no se dispone de servicios de salud o en los hospitales y puestos de salud sólo atienden a los que tienen plata10(5).

Así, el Cric va construyendo la naciente política indígena que se transformará en el Programa de Salud creado en el sexto congreso en 1981, primera institución propia regional de salud con una plataforma política ampliada: Fortalecimiento político organizativo que promoverá una institucionalidad propia descentralizada como gobierno en salud; 2) Relaciones con el Estado, donde el Cric como sujeto político con voz propia en salud interlocuta, exige y negocia; 3) Fortalecimiento de la medicina tradicional como articulador de la política indígena de salud mediante médicos tradicionales, sob anderos, pulseadores y parteras para armonizar el territorio, las autoridades, las familias y acompañar espiritualmente las luchas por tierra; 4) Acompañamiento a pacientes con personal bilingüe para garantizar su acceso sin discriminación a servicios de salud: El Indio no está solo; 5) Formación de personal propio: Agentes Comunitarios para promover la medicina tradicional y el uso de plantas, prestar atención básica de medicina occidental y mediar entre el sistema médico occidental y la medicina propia y que fortalezcan la organización en salud.

En medio de la represión sistemática del Estatuto de Seguridad del gobierno Turbay (78-82) y enfrentando el Estatuto Indígena, que buscaba acabar los resguardos y los cabildos, el Cric, y otras organizaciones regionales propician la unidad indígena que se concreta en la creación de la Organización Nacional Indígena de Colombia (Onic) en 1982 que, en adelante, articulará la resistencia y las luchas a nivel nacional e internacional.

En 1981, el Estado implementa el Sistema Nacional de Salud (SNS) con un subsector de asistencia pública para pobres (incluidos los indígenas), uno de seguridad social para trabajadores y uno privado para población con capacidad de pago en un contexto de transición 
del discurso preventivista de la triada ecológica de los sesenta ${ }^{12}$, al de derechos humanos de Atención Primara de Alma-Ata (1978).

No obstante, el talante represivo del gobierno Turbay, el Ministerio de Salud expide en septiembre de 1981 la Resolución 10.013, luego de 6 meses de creado el Programa de Salud del Cric, en medio de las exigencias indígenas de una política de salud especial y en el contexto internacional del reconocimiento de la 'otredad con derechos'.

La 10013, hito contencioso de la política de salud indígena del Estado, da lugar al reconocimiento de la otredad en los límites del pensamiento colonial, pues reconoce la existencia de visiones y prácticas de un otro pero como míticas y subordinadas a la autoridad sanitaria del Estado y la hegemonía del conocimiento científico. En materia de financiamiento declara la gratuidad de los servicios de salud, sin alusión a la capacidad de pago en un virtual reconocimiento de derechos. Así lo destaca la Onic:

[...] se convierte en el primer espacio legal de lucha, en el que se trabaja por primera vez la gratuidad, como un derecho económico para quienes su organización productiva-económica implica reciprocidad, redistribución y complementariedad, no mercado y dinero, aunque tienen relación con los dos ${ }^{13(126)}$.

La 10013 crea la figura de promotores en referencia al personal propio y, de esta forma, los Agentes Comunitarios del Cric, aunque con perfil similar,desaparecerán para convertirse en promotores indígenas con menor autonomía política y terapéutica, debido a su progresiva dependencia laboral y técnica del Estado.

Los años 80 transcurrían entre el ajuste estructural, la exclusión política, la movilización social y los esfuerzos de paz. Mientras, en el Cauca, se posicionaba el grupo armado indigenista Quintín Lame, sin aprobación del Cric, para enfrentar el ataque a las luchas indígenas Finalizando el gobierno Barco (86-90) y empezando el gobierno Gaviria (90-94) en plena apertura económica, se concretan acuerdos de paz con las guerrillas del M-19, el Quintín Lame y el EPL con impacto sobre el proceso constituyente.

En este marco, en 1986, la Onic crea el Programa de Salud, incorporando los desarrollos del Cric para proyectarlos a nivel nacional y reforzar la incidencia política frente a la exigibilidad de la 10013.

En 1989, se produce el convenio 169 de la Organización Internacional del Trabajo (OIT), incorporado por Colombia mediante la Ley 21 de 1990 que supera el asimilacionismo del convenio 107 de 1957:

Eliminar la orientación hacia la asimilación de las normas anteriores; Reconociendo las aspiraciones de esos pueblos a asumir el control de sus propias instituciones y formas de vida y de su desarrollo económico y a mantener y fortalecer sus identidades, lenguas y religiones, dentro del marco de los Estados en que viven ${ }^{14(16)}$.

Se instaura, así, la consulta previa e informada, el reconocimiento de la gobernabilidad de los pueblos indígenas sobre sus formas de vida y sus instituciones como núcleo duro jurisprudencial y político a nivel global y de respaldo a las luchas generales y en salud de los pueblos indígenas.

En 1990, se expide la Ley 10 de 1990 que inicia el tránsito del SNS a la privatización, a través de la intermediación administrativa y financiera de los servicios de salud y la vinculación del derecho a la salud a la capacidad de pago. La demanda de cumplimiento de la 10.013, el ascenso de las luchas indígenas y el acceso al gobierno de sectores progresistas en el marco de los acuerdos de paz, llevaron a la expedición del Decreto 1.811 de 1990 que,no obstante, el discurso del reconocimiento, mantiene el tono integracionista de la 10.013 e incentiva la cofinanciación comunitaria de los servicios como prólogo a la privatización de los mismos. La 10.013 y el 1.811 son apropiadas como instrumentos de lucha del Cric para 
enfrentar la discriminación y/o la negación de servicios de salud y para fortalecer su agenda propia de salud.

Estos primeros 20 años marcan la emergencia del sujeto político indígena en el Cauca y en Colombia sobre arenas contenciosas que combinan el reconocimiento estatal con la racialización violenta de aquello que desestabiliza su estructura de poderes y saberes hegemónicos. En salud, el Cric sentó las bases de una política indígena de salud con instituciones de gobierno (Cabildos y Programa de Salud), normas y mandatos (Plataforma en salud), saberes (Medicina tradicional y atención intercultural), agentes propios (sabedores ancestrales), formación e investigación y modelo propio e intercultural basado en la cosmovisión, la ley de origen, el derecho mayor y el derecho propio. Mientras, el Estado, respondiendo al contexto internacional y a la movilización indígena, inició la política de salud indígena con la 10.013 y el 1.811.

\section{La Constitución de 1991: Visibilidad estructural del sujeto político indíge- na (1990-1991)}

En el marco de las negociaciones con la insurgencia, emerge un movimiento ciudadano por la paz y la inclusión política. En las elecciones de mayo de 1990 este movimiento se autoconvoca a votar por una Asamblea Nacional Constituyente (ANC). En diciembre del mismo año se eligen los Constituyentes. En este proceso son electos dos indígenas en contienda abierta y uno más a partir de los acuerdos de paz con el Quintín Lame.

La nueva Constitución Política (nCP) proclamó el Estado Social de Derecho, la función social y ambiental de la propiedad privada, supero el clericalismo, rodeó de garantías la participación democrática y dejó abiertas las puertas para el modelo neoliberal. Es un Estado multicultural que reconoce la diferencia en clave de derechos, recoge las corrientes internacionales de la discusión étnica, que tramita la 'otredad' en una nueva fase del integracionismo vía mercado. Los pueblos indígenas ahora son sujeto político de derechos y parte del ordenamiento Estatal a nivel territorial y político"1.

El derecho a la salud quedó definido en la nCP como servicio público de carácter prestacional para la promoción, protección y recuperación de la salud a brindarse por públicos y privados en apertura hacia la privatización de la salud y la seguridad social'1 ${ }^{11}$. Aunque no se menciona la salud indígena, el reconocimiento constitucional de la diversidad étnica y cultural de la nación coloca en un nivel de mayor jerarquía el proceso contencioso entre políticas indígenas y estatales en salud ${ }^{1}$.

\section{De la Ley 100 a la 691: nuevas invisi- bilidades e inclusiones vía mercado (1993-2001)}

La euforia constituyente terminó con el asesinato de 20 indígenas Nasa en el Nilo-Cauca, en diciembre de 1991, a manos de fuerzas estatales penetradas por el narcotráfico, iniciando una nueva etapa de violencia contra los pueblos indígenas y de resistencia y organización del Cric.

En salud, el gobierno expide de manera inconsulta la Resolución 5.078 en 1992 que reduce la medicina tradicional a terapéutica y las culturas médicas a cúmulo de conocimientos y prácticas,con lo que se limitaría la disputa intercultural de saberes y poderes en un tema nuclear para los sistemas de salud. En materia de gobierno, administración y financiamiento, el gobierno expide el decreto 1088 de 1993 que define las Asociaciones de Cabildos como entidades públicas de carácter especial que podrán manejar recursos públicos, fortaleciéndose el doble rol indígena como movimiento social y autoridad político-administrativa territorial. Esta norma parcial posponía el compromiso constitucional de crear entidades territoriales indígenas y no respondía a lo concertado con los pueblos indígenas.

En diciembre de 1991, se expide de manera inconsulta con los pueblos indígenas la Ley 100, que reformó el SGSSS inspirada en el plan 
de ajuste derivado del consenso de Washington en materia de disciplina fiscal, reorientación de subsidios, privatización y desregulación económica a favorecer de la libre competencia'.

Esto trajo la intermediación administrativo-financiera para la gestión del riesgo financiero y de salud en un esquema de subsidios a la demanda y de aseguramiento individual con una Unidad de Capitación (UPC) y un Paquete Obligatorio de Salud (POS) diferenciado y subsidiado para pobres sin capacidad de pago y contributivo para aportantes, en una versión del modelo neoliberal denominada pluralismo estructurado:

'Pluralismo' evita los extremos del monopolio en el sector público y la atomización en el sector privado, 'Estructurado' evita los extremos de los procedimientos autoritarios del gobierno y la ausencia anárquica de reglas del juego transparentes para evitar o compensar las fallas del mercado ${ }^{\mathbf{1 5}(16)}$.

Para la Ley 100 los indígenas eran pobres sin capacidad de pago y con derecho a crear empresas administradoras, lo que obligó el tránsito de la gratuidad (10.013 y 1.811) hacia subsidios parciales para demandar servicios de salud occidentalesviolándose el derecho a la consulta libre e informada. Aunque el Cric rechazó la ley, las empresas privadas ingresaron con engaños y sin consentimiento a los territorios indígenas a afiliar población,generando confusión por los beneficios ofrecidos y limitando una respuesta indígena unificada.

En 1995, miles de indígenas se congregan en el Resguardo de la María, Piendamó, en el Cauca, convocados por el Cric. Los indígenas protestan contra el gobierno por el incumplimiento de acuerdos sobre tierras, educación, derechos humanos y,en salud, exigen la salida de la Ley 100 y las empresas, así como concertar un sistema especial de salud. El gobierno descartó reformar el SGSSS, salvo ajustes básicos de normatividad indígena lo que, sumado a la confusión entre gratuidad y subsidio, lleva a que en una nueva movilización en 1996 (Novirao), el Cric acepte transitoriamente entrar en el SGSSS con adecuaciones socioculturales, mientras se concierta su propuesta de un Régimen Especial Indígena en Salud (Reis) por fuera de la Ley 100.

Esto llevó a una primera etapa de modulaciones del SGSSS que, entre otros, estableció la afiliación colectiva a cargo del cabildo, eliminación de copagos y cuotas moderadoras para hacer equiparable 'subsidio a gratuidad', prohibición de mercadeo en territorios indígenas y creación de empresas y prestadores indígenas de salud, financiando, incluso como acuerdo de Novirao, un estudio para la estructuración de la empresa del Cric. Con ello avanzaba, de manera contenciosa, la era de inclusión y reconocimiento vía mercado.

En 1997, el Décimo Congreso del Cric aprueba crear una empresa de salud sin abandonar la exigencia del Reis y transita por el camino de aprovechar lo posible/impuesto y de construir lo propio. En diciembre del mismo año nace la Asociación Indígena del Cauca (AIC), empresa Administradora del Régimen Subsidiado de Salud Indígena (Arsi), iniciando un camino de empresarización en disputa entre las exigencias de mercado y las del modelo médico hegemónico, con la garantía del derecho a la salud intercultural y se crean, progresivamente, Instituciones Prestadoras de Servicios de Salud Indígenas (Ipsi), para servicios de medicina occidental con posibilidad de adecuaciones socioculturales. Se crean Arsi en todo el país, afiliando a cerca del 80\% de los indígenas y surgen más de 100 Ipsi, de las cuales 8 son del Cauca. Estas, bajo la dirección del Programa de Salud, desafían la lógica empresarial de la AIC para incorporar elementos de política propia dentro del SGSSS.

Los años 90 terminan con un fuerte desarrollo neoliberal, la explosión del desplazamiento forzado por la violencia y un nuevo proceso de paz del Gobierno Pastrana (1998-2002) con las Fuerzas Armadas Revolucionarias de Colombia (Farc). El incumplimiento de los acuerdos suscritos por los gobiernos con los pueblos indígenas lleva al Cric a declararse 
en 'emergencia social, económica y cultural' y movilizarse en 'Minga' de nuevo al resguardo de la María y suscribir un nuevo acuerdo con el gobierno convertido en decreto 982, que prometía cumplir lo incumplido y afrontarla emergencia. Ya en 1995 la Onic, mediante latoma de la conferencia episcopal, obtuvo la creación por decreto de la Mesa Permanente de Concertación (MPC). Uno y otro instrumento terminarán siendo ineficaces, advirtiéndose una impronta del régimen político que combina exclusión y racismo. En salud termina el periodo con adaptaciones parciales del SGSSS y exigencias de un Reis, mientras que el Cric, bajo un modelo de gobierno colectivo,transita por la disputa entre mercado, derechos y desarrollo de modelos propios de salud.

El Cric y la Onic dejan registrado en sus congresos el rechazo al SGSSS y su opción por sistemas propios e interculturales de salud. El Programa de Salud del Cric enfrenta un debilitamiento de su rol en razón a que los componentes de la política indígena de salud pasan a ser asumidos por la AIC y las Ipsi,con esfuerzos destacados para construir modelos de salud interculturales, pero limitados por las lógicas de la Ley 100.

Adicionalmente el SGSSS acabó con los promotores de salud, convirtiéndolos en técnicos de salud para administración y demanda inducida de pacientes, con lo que el Cric perdió influencia en su formación. Este es un punto de inflexión que impacta las estructuras indígenas de salud para la construcción de la política propia, pero que no detendrán su propósito.

\section{2-2010 - Seguridad democrática y emergencia del Sispi}

Iniciando el 2002, se rompen los diálogos gobierno-Farc y es elegido presidente Uribe Vélez. Su política, denominada de Seguridad Democrática, estigmatiza las luchas indígenas, desconoce el derecho a la consulta libre e informada frente a actividades extractivas y la ocupación territorial por la fuerza pública y descalifica los acuerdos suscritos por gobiernos anteriores. Esto implica 8 años de un escenario de violaciones de DDHH. Sin embargo, el Plan Nacional de Desarrollo (PND) 2006-2010 de Uribe fue declarado inexequible por la Corte Constitucional por no consultar los pueblos indígenas.

El Cric participa en 2005 en Cuenca Ecuador en la II Asamblea Mundial por la salud de los Pueblos. En esta asamblea da a conocer sus luchas, comparte experiencias desde la interculturalidad y suscribe la declaración por la resonancia en temas como:

El MSP incorporará temas clave como la lucha contra los Tratados de Libre Comercio, reforma agraria y restauración de la tenencia de la tierra a los pueblos indígenas, protección contra el saqueo del conocimiento ancestral como una defensa fundamental de la seguridad social, identidad cultural y seguridad nutricional $[\ldots]^{16(3)}$.

Con ello se mantiene la vocación de movilización e incidencia global, ya demostrada frente a la Organización Panamericana de la Salud (OPS) en Winnipeg en 1993 y en la movilización continental contra el mal llamado descubrimiento de América en 1992.

El gobierno Uribe bombardea la minga indígena de 2006 en la María Piendamó, incluida la misión médica; mientras militariza resguardos, recluta jóvenes indígenas y se abstiene de votar la declaración universal de DDHH de los pueblos indígenas de Naciones Unidas,en abierta contradicción con la jurisprudenciavigente, $\mathrm{y}$ reeditando el racismo de la contienda entre régimen político y pueblos indígenas por lo que nuevamente el Cric, en protesta, se moviliza a finales del año en un hecho que cobró vidas indígenas a manos de la fuerza pública,. En 2009 el gobierno adoptó la Declaración en mención y el Cric y el movimiento indígena se posicionó como sujeto político desafiante y alternativo, elevando el costo político al régimen por su talante autoritario.

La década transcurre para el tema de salud en medio de una crisis de legitimidad, 
operatividad y sostenibilidad financiera del SGSSS que el gobierno intenta paliar en 2010 con la declaratoria de una emergencia social, que en realidad profundizaba el modelo privatizador. A partir de allí la presión social, incluida la indígena, y la revisión de la Corte Constitucional la tornan inexequible. En estos años la disputa se concentra en adecuaciones del SGSSS, para funcionalizar la operación de las empresas y prestadoras indígenas, persistiendo la tensión sobre la necesidad de un sistema propio que se expresa en la primera Asamblea Nacional de Salud de los pueblos indígenas en Villeta ${ }^{17}$, donde se cuestiona el SGSSS:

[...] bajo [...] la Ley 100 de 1993, los pacientes pasamos a ser clientes, convirtiendo el derecho a la salud en una mercancía o negocio que favorece los intereses privados [...] [lo cual requiere] [...] reformas estructurales que parten de la derogación de la Ley 100 y de su modelo perverso que ha fracasado en nuestro país ${ }^{\mathbf{1 7 ( 1 )}}$.

Se denuncian los incumplimientos del Gobierno para la adecuación sociocultural y la atención precaria a comunidades indígenas en varios departamentos que llevan a la Asamblea a adoptar propuestas del Cric, la Onic, el Consejo Regional Indígena de Caldas (Cridec) y el pueblo Arhuaco, en el sentido de construir el Sispi como alternativa al SGSSS, a las Empresas Promotoras de Salud Indígena (Epsi) (Anteriormente llamadas Arsi) y a las Ipsi.

\section{0-2017 - Las luchas por el Sispi en tiempos de construcción de la Paz}

Los gobiernos de Santos (2010-2018) avanzan la modernización neoliberal del Estado que continúa la agenda precedente con una apertura del régimen político orientada a hacer la paz con las Farc y el Ejército de Liberación Nacional (ELN). Por primera vez un gobierno consulta el Plan Nacional de Desarrollo con los pueblos indígenas, aunque persisten las agresiones contra las comunidades y el territorio y el incumplimiento de los acuerdos.
Esto intensifica la movilización indígena y las recuperaciones de tierras, llamadas ahora 'Liberación de la Madre Tierra' que, más que recuperar, es liberar el territorio del desarrollo depredatorio para dar paso a su cuidado como expresión de equilibrio y salud en una perspectiva contracivilizatoria.

La crisis del SGSSS se enfrenta a microreformas operativas que mantienen el pluralismo estructurado en fase avanzada de privatización, eliminación de las empresas públicas, salvo las indígenas que son de carácter especial, y destrucción de la red pública hospitalaria que atiende las zonas rurales, donde no hay rentabilidad para los privados. En salud indígena, el SGSSS, luego de varios años, asignó parcialmente recursos para los procesos de adecuación sociocultural a cargo de las empresas indígenas de salud (Epsi); en 2013 se creó la Subcomisión Nacional de Salud Indígena, instancia mixta (gobierno-indígenas) de concertación como parte de la MPC y, en 2014, se expide el Decreto 1.953 (hitó más representativo del periodo) a fuerza de movilización en el Cauca y en el país, en el que de manera provisional, a cambio de crear las entidades territoriales indígenas, se adopta el esquema de funcionamiento de los territorios indígenas para la administración directa de recursos públicos y de los Sistemas Propios e Interculturales de Educación (Seip), de salud Sispi y Justicia entre otros.

El Sispi, desde los pueblos indígenas, es el ejercicio de la política indígena en salud en diálogo con la política de salud indígena del Estado contenida dentro del SGSSS y otras políticas públicas, pero en términos del decreto:

[...] El Sispi se articula, coordina y complementa con el Sistema General de Seguridad Social en Salud, SGSSS, con el fin de maximizar los logros en salud de los pueblos indígenas $18(1)$

Con ello establece una relación de complementariedad y de subordinación práctica a la Ley 100 que copa el espacio real de disputa 
de poderes y saberes de la interculturalidad realmente existente.

El Sispi, desde los pueblos indígenas, contiene 5 componentes que dan continuidad a los ejes de la política indígena de salud desde el nacimiento del programa de salud del Cric en 1981 y de la Onic en 1986: 1) Político organizativo para relaciones con el Estado, gobierno en salud, desarrollo de la ley de origen, derecho mayor y derecho propio, para cuidar la vida y el territorio; 2) Administración-Gestión desde la ancestralidad para manejo de recursos económicos,información, personal de salud e información en coordinación con la administración pública y privada; 3) Formación e Investigación para la reproducción de capacidades técnicas, políticas y socioculturales del personal propio y para la recuperación, producción y difusión de saberes y su relación intercultural con otras culturas; 4) Cuidado de la salud que organiza saberes, prácticas y caminos para la armonía individual, familiar y comunitaria desde articulación contenciosa de la sabiduría ancestral, las medicinas alternativas y occidental; 5) Sabiduría ancestral que orienta espiritualmente todo el Sispi.

Este decreto traza nuevos límites a la disputa política entre pueblos indígenas y Estado en las que la tensión entre lo individual y lo colectivo, el buen vivir y el desarrollo y lo público y lo privado, exige la reforma al SGSSS para que el Sispi se materialice.

En 2015 se expide la Ley Estatutaria en Salud que declara la salud como derecho fundamental autónomo y exigible y por la incidencia del movimiento indígena. Establece que

Para los pueblos indígenas el Estado reconoce y garantiza el derecho fundamental a la salud integral, entendida según sus propias cosmovisiones y conceptos, que se desarrollan en el Sistema Indígena de Salud Propio e Intercultural (Sispi)19(4).

Con lo que se amplía y eleva la jerarquía normativa que soportan luchas indígenas por el derecho a la salud.
En 2017 el Cric registra poco avance en el Sispi, por lo que en su XV congreso define iniciar una etapa de transición en la que se transformarán la Epsi y la Ipsi en instituciones propias que fortalezcan el gobierno y los modelos propios en salud. Para tal efecto ordena “[...] iniciar el desmonte del modelo de salud de la Ley 100, el cual debía dejar de regir en el Cauca indígena en un plazo de un año"4(85).

Meses después, en la gran Minga de Monterilla (carretera panamericana), $15 \mathrm{mil}$ indígenas del Cric protestan por los incumplimientos del gobierno y exigen el reconocimiento y apoyo a su mandato de transitar por el Sispi por fuera de la Ley 100.

\section{Conclusiones}

La irrupción del Cric cambió la historia de las relaciones entre los pueblos indígenas, la sociedad general y el Estado en Colombia al configurarse como un nuevo sujeto político de base territorial, con formas propias de identidad, autoridad y gobierno, economía y desarrollo sociocultural que desestabiliza la racialización de las relaciones asimétricas de poder y saber coloniales y contracivilizatorios. Este proceso entre, 1971 y 2017, se inscribe en una contienda entre régimen político excluyente, racista y de pensamiento colonial y luchas por la participación democrática; entre desarrollo y buen vivir y entre mercado $\mathrm{y}$ derechos humanos.

La configuración de la política de salud indígena ha resultado de una disputa permanente y cambiante entre pueblos indígenas y Estado que se ha traducido en estructuras institucionales, normativas y programáticas dinámicas a nivel estatal en el SGSSS de pluralismo estructurado privatizado y con adecuaciones socioculturales fruto de resistencia y movilización indígena y, nivel del Cric, que ha construido una política indígena de salud propia e intercultural de salud.

La política de salud indígena, desde la Resolución 10.013, ha pasado del 
integracionismo de corte colonial, pasando por el enfoque de derechos del 1.811 al multiculturalismo de los derechos de la constitución y se ha transmutado en la inclusión subordinante del modelo neoliberal de la Ley 100 y todos sus desarrollos y adecuaciones socioculturales incluida la Ley 691.

La lucha por el derecho fundamental a la salud en una perspectiva intercultural, ha implicado un proceso permanente de desestabilización y alteración de las asimetrías de poderes y saberes entre el modelo hegemónico de desarrollo depredador de la vida y el territorio, la medicina occidental y democracia restringida y un modelo orientado hacia el buen vivir, la sabiduría ancestral y la acción política colectiva basada en la ley de origen, el derecho mayor y el derecho propio de los pueblos indígenas.

\section{Colaborador}

Urrego-Rodríguez JH(0000-0001-5972-7595)* es responsable de redactar el manuscrito.

\section{Referencias}

1. Quijano A. Colonialidad del poder y clasificación social. En: Castro-Gómes S, Grosfoguel R, editores. El giro decolonial. Reflexiones para una diversidad epistémica más allá del capitalismo global[internet]. Bogotá, DC: Siglo del Hombre; 2007. [acceso en 2019 mayo 20]. Disponible en: http://www.unsa.edu.ar/ histocat/hamoderna/grosfoguelcastrogomez.pdf.

2. Walsh C. Interculturalidad y Colonialidad del Poder. Un pensamiento y posicionamiento "otro" desde la diferencia colonial. En: Castro-Gómes S, Grosfoguel R, editores. El giro decolonial, Reflexiones para una diversidad epistémica más allá del capitalismo global [internet]. Bogotá: Siglo del Hombre; 2007. [acceso en 2019 mayo 20]. Disponible en: http://www. unsa.edu.ar/histocat/hamoderna/grosfoguelcastrogomez.pdf.

Colombia. Ministerio de Salud y Protección Social. Perfil de Salud de la Población Indígena, y medición de desigualdades en salud Colombia 2016 [internet]. Bogotá, DC: MinSalud;2016. [acceso en 2019 mayo 20]. Disponible en: https://www.minsalud.gov.co/sites/rid/Lists/BibliotecaDigital/RIDE/VS/ED/PSP/ Perfil-salud-pueblos-indigenas-colombia-2016.pdf.

4. CRIC, Urrego J. Avance en la construcción del Sistema Intercultural en Salud Propia e Intercultural-SISPI. Popayán: Samava Ediciones; 2018.

5. Colombia. Ley 89. 25 de noviembre de 1890. Por la cual se determina la manera como deben ser gobernados los salvajes que vayan reduciéndose a la vida civilizadaEl congreso de Colombia. Diario Oficial. 25 Nov 1890.

6. Colombia. Departamento Nacional de Planeación. Los pueblos indígenas de Colombia en el umbral del nuevo milenio. Población, cultura y territorio: bases para el fortalecimiento social y económico de los pueblos
${ }^{*}$ Orcid (Open Researcher and Contributor ID). 
indígenas[internet]. Bogotá, DC: DNP; 2004. [acceso en 2019 mayo 20]. Disponible en: https://www.dnp. gov.co/programas/desarrollo-territorial/Paginas/ pueblos-indigenas.aspx.

7. Archila M, García M, Parra L, et al. Cuando la copa se rebosa, luchas sociales en Colombia 1975-2015. Bogotá, DC: CINEP; 2018.

8. Centro de Investigación y Educación Popular. Cómo nació el CRIC, primeras luchas en Congreso Consejo Regional Indígena del Cauca-CRIC, 10 años de luchas, historia y documentos. Rev. Controversia. 1992; (91-92):11.

9. Gros C, Morales T. ¡A mi no me manda nadie!:Historia de Vida de Trino Morales. Instituto Colombiano de Antropología e Historia. Bogotá, DC: Editorial Perfiles; 2009.

10. Consejo Regional Indígena del Cauca. Las comunidades indígenas en el paro cívico nacional. Unidad Indígena. Unidad, Territorio, Cultura y Autonomía. Popayán: CRIC; 1977.

11. Urrego J. Configuración de la política de salud indígena en Colombia 1971-2017. [tese]. Bogotá, DC: Universidad Nacional de Colombia; 2019.520 p.

12. Breilh J. La determinación social de la salud como herramienta de transformación hacia una nueva salud pública (salud colectiva). Rev. Fac. Nac. Salud Pública [internet]. 2013 [acceso en 2019 mayo 18]; 31(supl1):13-27. Disponible en: http://www.scielo. org.co/pdf/rfnsp/v31sl/v31sla02.pdf.

13. Organización Nacional Indígena de Colombia. Memorias del V Congreso Nacional Indígena. Los pueblos Indígenas de Colombia. Un reto hacia el nuevo milenio[internet]. Bogotá, DC: Fundación Etnollano; 1998. [acceso en 2019 mayo 18]. Disponible en: http://observatorioetnicocecoin.org.co/cecoin/files/Memorias\%20del\%20quinto\%20congreso\%20 nacional\%20indigena.pdf.

14. Organización Internacional del Trabajo. Convenio Num. 169 de la OIT sobre Pueblos Indígenas y Tribales [internet]. Bogotá, DC: OIT; 2014. [acceso en 2019 jun 2]. Disponible en: https://www.ilo.org/wcmsp5/ groups/public/---americas/---ro-lima/documents/ publication/wcms_345065.pdf.

15. Londoño JL, Frenk J. Pluralismo Estructurado: Hacia un Modelo Innovador para la Reforma de los Sistemas de Salud en América Latina [internet] Washington: BID; 1993. [acceso en 2019 mayo 29]. Disponible en: https://publications.iadb.org/publications/spanish/ document/Pluralismo-estructurado-Hacia-un-modelo-innovador-para-la-reforma-de-los-sistemas-de-salud-en-Am\%C3\%A9rica-Latina.pdf.

16. Movimiento por la Salud de los Pueblos. Declaración de la II Asamblea Mundial de salud de los Pueblos [internet]. 2015. [acceso en 2019 mayo 16]. Disponible en: https://web.ua.es/opps/objetivos_del_desarrollo_del_milenio/ponencias/martes_manyana_47_ presentacion.pdf.

17. Consejo Regional Indigena Del Cauca. Pronunciamiento final de la Asamblea Nacional de Salud de los Pueblos Indígenas. Construyendo alternativas para el buen vivir desde los pueblos[internet]. Villeta: CRIC; 2010. [acceso en 2019 mayo 16]. Disponible en: https://www.cric-colombia.org/portal/pronunciamiento-final-de-la-asamblea-nacional-de-salud-de-los-pueblos-indigenas-construyendo-alternativas-para-un-buen-vivir-desde-los-pueblos/.

18. Colombia. Ministerio del Interior. Decreto 1.953 de 07 de Octubre de 2014. Por el cual se crea un régimen especial con el fin de poner en funcionamiento los Territorios Indígenas respecto de la administración de los sistemas propios de los pueblos indígenas hasta que el Congreso expida la ley de que trata el artículo 329 de la Constitución Política. Diario Oficial Oct2014.

19. Colombia. Ley Estatutaria en Salud 1751 de 16 de Febrero de 2015. Regula el derecho fundamental a la salud y se dictan otras disposiciones. Diario Oficial. 16 Feb2015.

Recebido el 24/06/2019

Aprovado el 08/12/2019

Conflicto de intereses: inexistente

Apoyo financiero: no hubo 